\title{
Geometrical determinations of IMRT photon pencil-beam path in radiotherapy wedges and limit divergence angle with the Anisotropic Analytic Algorithm (AAA)
}

\author{
Francisco Casesnoves \\ Society for Industrial and Applied Mathematics (SIAM), Denver, Colorado, USA.
}

Received March 13, 2014; Revised May 08, 2014; Accepted May 10, 2014; Published Online May 18, 2014

\section{Original Article}

\begin{abstract}
Purpose: Static wedge filters (WF) are commonly used in radiation therapy, forward and/or inverse planning. We calculated the exact 2D/3D geometrical pathway of the photon-beam through the usual alloy WF, in order to get a better dose related to the beam intensity attenuation factor(s), after the beam has passed through the WF. The objective was to provide general formulation into the Anisotropic Analytical Algorithm (AAA) model coordinates system (depending on collimator/wedge angles) that also can be applied to other models. Additionally, second purpose of this study was to develop integral formulation for 3D wedge exponential factor with statistical approximations, with introduction for the limit angle/conformal wedge. Methods: The radiotherapy model used to develop this mathematical task is the classical superposition-convolution algorithm, AAA (developed by Ulmer and Harder). We worked with optimal geometrical approximations to make the computational IMRT calculations quicker/reduce the planning-system time. Analytic geometry/computational-techniques to carry out simulations (for standard wedges) are detailed/developed sharply. Integral developments/integral-statistical approximations are explained. Beam-divergence limit Angle for optimal wedge filtration formulas is calculated/sketched, with geometrical approximations. Fundamental trigonometry is used for this purpose. Results: Extent simulation tables for WF of $15^{\circ}, 30^{\circ}, 45^{\circ}$, and $60^{\circ}$ are shown with errors. As a result, it is possible to determine the best individual treatment dose distribution for each patient. We presented these basic simulations/numerical examples for standard manufacturing WF of straight sloping surface, to check the accuracy/errors of the calculations. Simulations results give low RMS/Relative Error values (formulated) for WF of 15, 30, 45, and 60 . Conclusion: We obtained a series of formulas of analytic geometry for WF that can be applied for any particular dose delivery model. Simulations results gave acceptable trigonometrical approximations/data that can be used for LINAC applications/planning-system software. The integral formulas presented are practical for dose delivery calculations/3D-approximations when using WF/other similar types of beam modification devices. Limit angle formulation and conformal wedge concept was also presented.
\end{abstract}

Keywords: Radiotherapy; Intensity Modulated Radiation Therapy; Static Alloy Wedges; Analytic Geometry; Treatment Planning Optimization (TPO); Analytic Anisotropic Algorithm (AAA); Superposition-Convolution Models (SCM)

\author{
Corresponding author: Francisco Casesnoves MSc (Physics) MD; \\ Computational Bioengineering Researcher, SIAM (Individual \\ Researcher), Denver, Colorado State, USA. \\ Email: mathematics8@gmx.com \\ Cite this article as: \\ Casesnoves F. Geometrical determinations of IMRT photon pen- \\ cil-beam path in radiotherapy wedges and limit divergence angle \\ with the Anisotropic Analytic Algorithm (AAA). Int J Cancer \\ Ther Oncol 2014; 2(3):02031. DOI: 10.14319/ijcto.0203.1
}

\section{Introduction}

Currently, radiotherapy treatment is based on two main objectives: (1) First, to deliver the optimal radiation at Planning Target Volume(s) (PTVs), and (2) secondly, to minimize the dose magnitude in the Organs at Risk (OARs), and surrounding PTV tissues. Therefore, the radiation delivery should conform the tumor shape with the most convenient approximation(s).

There are a number of reasons to consider conformal and optimized radiotherapy as the most important objective for cancer treatment when radiation is included into the general treatment planning of the disease. The optimal dose guaran- 
tees a standard level of life quality, and maximum statistical survival time for the patient. Survival time has recently been determined, with clinical trials, when the tumor treatment is a combination of methods (RT and Chemotherapy, usually). This fact implies that when the optimal dose distribution is reached, the most desirable pharmacological effect will appear in the PTV (direct) and OARs (inverse). The explanation is that PTVs tumor cells will be killed or seriously damaged by radiation prior to the drug's action, and just the inverse process occurs at OARs regions (that is, the less radiation on OARs, the better natural cell conditions to avoid/minimize side-effects of drugs).

The patient quality of life also depends on minimum damage within the OARs surrounding the tumor, otherwise basic physiological functions could be altered and/or suppressed (e.g., urethra wall destroyed in Prostate Cancer Radiation, or Spinal Cord serious malfunction when surrounding metastasis are radiated). The side-effects of radiation should be minimized for the best TPO. The stages of dose delivery should be set in such a way that the side-effects will be minimum while radiation efficacy should be optimal. Finally, the research for future TPOs depends on the application and performance of the best TPOs available, in order to obtain new research data to improve future radiation treatment, but starting from the most advanced previous research stage. Recently, new Radiation Therapy Advances adjutant to Chemotherapy have reduced mortality, increased the quality of life for the patient, decreased recurrences, metastasis, and, in specific cases, increased survival time. ${ }^{1,2}$ However, this assertion cannot be totally generalized because each cancer type shows type specific characteristics. ${ }^{3}$ The clear and statistically demonstrated advance is related to increment of quality of life and side-effects reduction, but not all the studies/trials carried out show a sharp increment of survival time and mortality reduction [refs]. In addition, Chemo-RT has been proven to reduce mortality compared to only Chemotherapy in important tumors in cancer epidemiology (e.g., clearly in breast cancer from the $2^{\text {nd }}-3^{\text {rd }}$ year after the tumor diagnosis-treatment, Figure 1).Medical technology advances responsible of these improvements are principally the significant increase of the radiation quality (provided by the engineering and physical design of the newest accelerators), and the development of new physical and computational radiation therapy methods. ${ }^{4}$ Among them, IMRT, IMPT (we refer to the specific Bragg-Peak dose deposition for protons which make it easier to conform to the tumor 3D shape and to control the distributed dose compared to photons), and the new generations of planning systems. Recently 5, an Analytical Proton Beam Model for proton dose calculations has been developed. This model is based on optimization of exponentials, a Gaussian term, and a power expansion of an initial Proton Stopping-Power Formula. Variational
Optimization and Monte-Carlo GEANT4 computational work is also used for model fitting. In addition, the new planning systems implement several options of Inverse Treatment Planning Optimization Algorithms by a group of distinct formulas, according to the running time available for the RT Planner. Computing power has experienced a significant growth in functional development in recent times ${ }^{6}$, a fact which has produced direct applications in RT specifically and Medical Technology in general. In addition, Large-Scale Computation and Software, High Performance Computing, future Exascale software/hardware, and recent Mathematical advances in computer science to provide different tools in a short time for industry and research labs, play an important role in these cutting-edge improvement stages. The particular field of Mathematical Methods and Modeling which has been developed in the last few decades, and the advances in Approximation Theory, are useful to simplify and create faster and more precise software in RT. Complementing this, the new mechanical engineering design of current RT apparatus (Linear Accelerators, LINACs) has resulted in a better IMRT beam/dose distribution (e.g. collimators, output windows, internal filters, rotation mechanics of couch and gantry, electronics, motors, gantry accessories, etc).

Nevertheless, apart from the principal mechanical and physical tools and parts of the new accelerators, the complementary use of Beam Modification Devices (BMDs) constitute an effective and easy way to get conformal dosage, without excessive technical effort, and waste of treatment time. The inconvenient of the BMDs is the dose distribution alteration, due mainly to the increment of scattering photons (more frequent in Kilovoltage than Megavoltage). BMD types are classified by Shielding, Compensation, Wedge Filtration and Flattering. There are a number of BMDs, among them, those most frequently used are Multileaf Collimators (MLC), Shielding, Compensators, Wedge Filters, and Penumbra Trimmers. We focus this paper on Wedge Filters, which are used in general for relatively superficial tumors. Wedges can be classified as: Universal, Dynamic, Virtual, and Pseudo-Wedges. $^{7}$

The use of wedges is justified for practical, technical and economic reasons. High-quality alloy materials are not expensive, and the size of the wedges makes their handling, change and substitution easy. Manufacturing of wedge filter

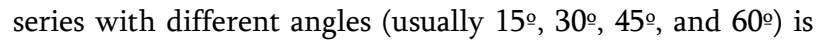
neither difficult nor expensive. Alloy is a high-endurance material which provides long industrial life for continuous RT sessions. In addition, several combinations of wedges in 2D and 3D configurations may be used to obtain a series of radiation distribution(s) to adapt the dose delivery to the tumor shape with accurate approximations. 


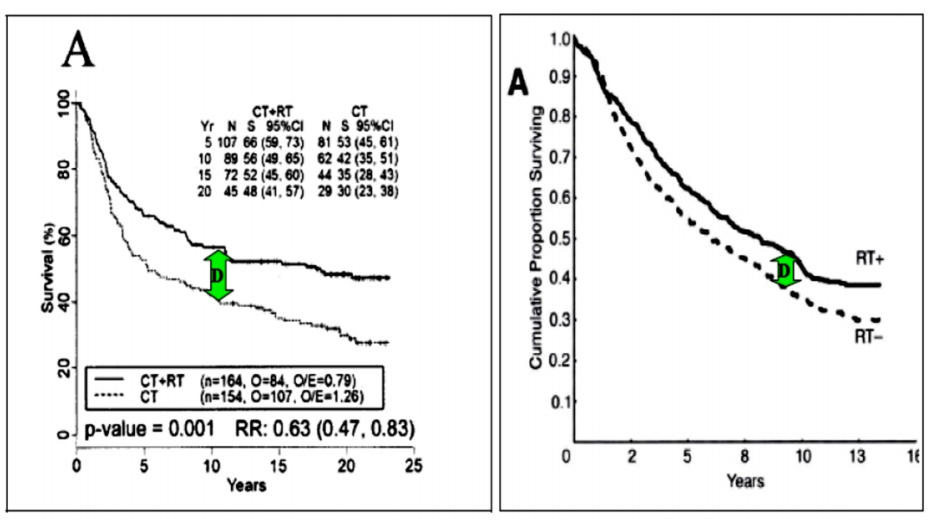

FIG. 1: Two significant trial-statistical results about survival time for Breast (left) and Rectal cancer (right) [Cancer Study of Ragaz \& Colleagues. ${ }^{2}$ The green arrows show the difference between the alternative treatments for both tumors. For Breast Cancer, the upper curve corresponds to the survival time with RT-Chemo, and the lower to only Chemotherapy. For Rectum tumors, the upper curve is for RT with Surgery, and the lower is for only Surgery. The difference of survival time is high from the approximately the $2^{\text {nd }}-3^{\text {rd }}$ year after diagnosis-treatment on.In breast cancer the difference of survival reaches, in some cases, a ratio higher than $20 \%$.

However, some of the approximations published do not take into account the specific 3D geometry of the wedge filter, and avoid developing a formal general-geometrical calculation based on a $3 \mathrm{D}$ planes intersection at an alpha angle (the proper wedge angle). Therefore, both exact and approximated calculations may be useful to add accuracy and simplicity to the planning software, through practical formulas. What is more, the wedge manufacturing can be designed and adapted to the specific conditions of each accelerator type. And also the materials could be improved to carry out a precise and clean attenuation and filtration of the beam, avoiding big dispersion, (contamination particles, mainly electrons, forward/backward scatter, and others).

It is useful and efficacious to generate knowledge about the different wedge options and combinations available to complement the treatment protocols; RT in general, External Beam Radiotherapy (EBRT), and IMRT. The direct practical result is a series of wedge options to be selected by planners according to the specific tumor types and shapes. The Coordinate System is a practical reference in TPO which can be used as general starting point to determine dose distribution and obtain equations to develop the coordinate/planning system. These geometrical formulas can be adapted or modified in IMTR for the different technical and mechanical options.

Therefore, the aim of this technical paper is to provide simple geometrical calculations and approximations for wedge use. We have developed the 2D/3D geometrical photon pencil-beam path through the wedge. The selected model is the AAA algorithm which integrates an attenuation factor into the integral formula, when wedges are used for conformal dose distribution. This exponential attenuation factor depends on the $3 \mathrm{D}$ pathway of the pencil beam through the wedge and its material. Secondarily, some approximations are derived from the principal exact formulas, whose objective is to provide practical mathematics for the planning system software (e.g., to avoid sine, cosine, complicated quadratic expressions, etc.). Limit Divergence Angle (LA) for optimal positioning of beam is defined, formulated, and sketched.

In brief, the objective of this contribution is practical and technical, in such a way that any RT researcher could develop and improve further formulation for other RT Models/Dose Delivery Systems. Finally, Simple Simulations are developed to determine error magnitudes, with complementary information about the mathematical development. Results obtained in a simulation series, e.g., for 30 wedges, show acceptable RMS errors $\left(\sim 10^{-2}\right)$ for the approximations carried out. Simulations are based on random values, and located at the positive quadrant (broad wedge part) of the wedge coordinate system.

\section{Methods and Materials}

\section{The Anisotropic Analytic Algorithm (AAA)}

The Analytic Anisotropic Algorithm, AAA, is a well-known and extensively used Superposition-Convolution Model in RT. AAA is is evolved from an initial Integral Superposition Convolution Model, whose parameters were optimized using large Monte Carlo experimental data in water. The starting Physical Equation to develop the model ${ }^{8-11}$ was a Yukawa Kernel based on the formulation structure of the classical Yukawa Gaussian Potential for Electromagnetism, as follows,

$D_{p}(r, z)=\sum_{k=1}^{3} \frac{C_{k}}{\sigma_{k}^{2}(z)} e^{-\frac{r^{2}}{\sigma_{k}^{2}(z)}}----(1)$

where $\mathrm{D}_{\mathrm{p}}(\mathrm{r}, \mathrm{z})$ the absorbed dose, normalized to one photon, $r$ is the radial coordinate

$r=\sqrt{x^{2}+y^{2}}$

in the transverse plane at depth $\mathrm{z}$. The characteristic func- 
$\mathrm{I}(\mathrm{z})$ denotes the area integral of the dose over the transverse plane of the pencil beam at depth $\mathrm{z}$, normalized to one photon, and $\sigma_{k}^{2}(z)$ is the mean square radial displacement of the profile at depth z. Next, a mathematical development based also in experimental data and Fourier Transform, was carried out ${ }^{8-11}$, to transform the initial formula on a triple sum of Gaussians (Superposition) from the initial simple Gaussian, and optimize the coefficients according to photon beam experimental data. As a result, the Pencil Model Dose at a depth $\mathrm{z}$ and into an almost differential cylinder (Triple Gaussian Pencil Beam) whose diameter is $2 r$ is,

$$
D_{p}(r, z)=I(z) \sum_{k=1}^{3} \frac{c_{k}}{\sigma_{k}^{2}(z)} e^{-\frac{r^{2}}{\sigma_{k}^{2}(z)}}---(2)
$$

The constants here are normalized in such a way that $\sum_{k=1}^{3} c_{k}=1$

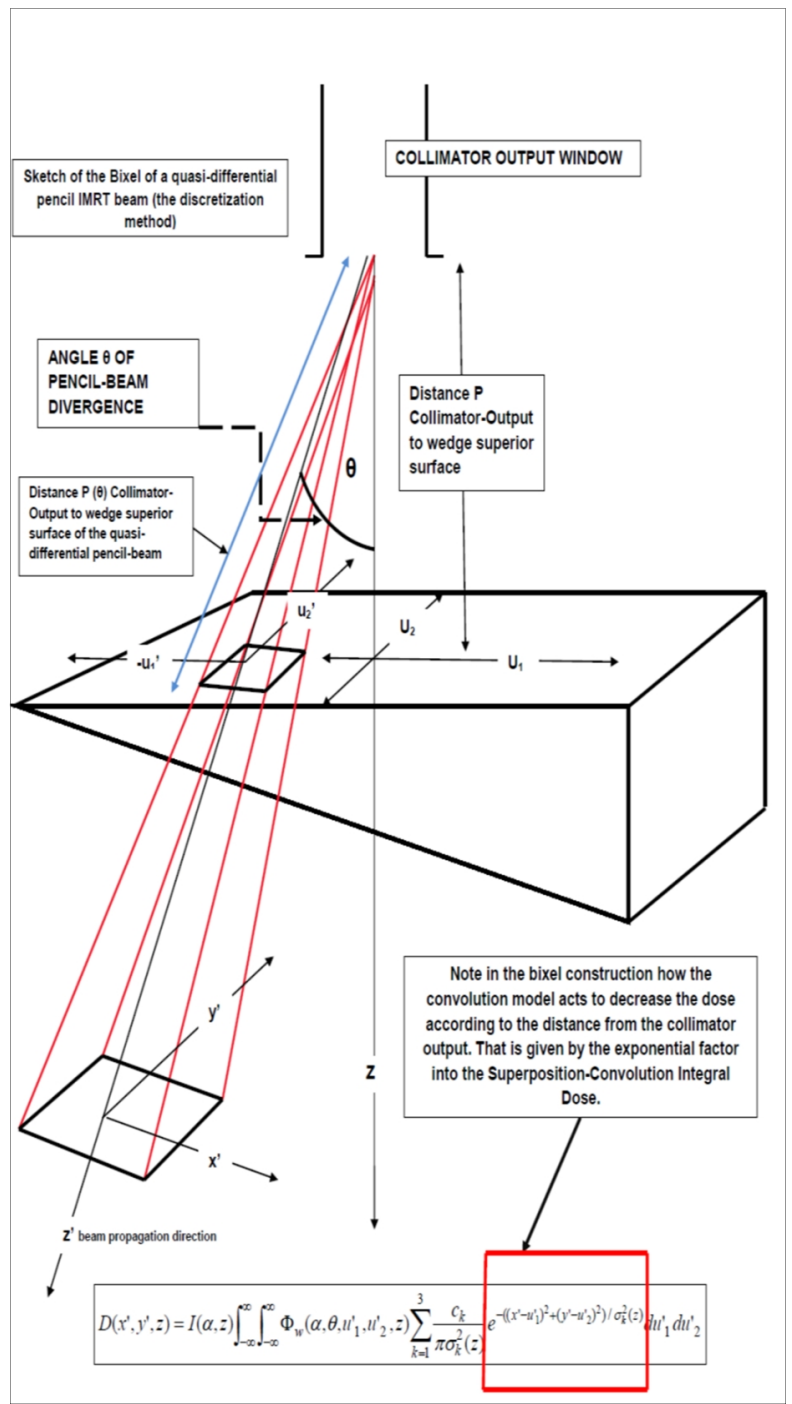

FIG. 2: Sketch of a bixel (a quasi-differential rectangular prism (a pyramid), whose apex is at collimator output) which contains a Pencil-beam whose path goes through the wedge with a divergence angle $\theta$. A Voxel is a quasi-differential portion of volume almost parallelepipedic). We detail complementary explanations about the Superposition-Convolution method to introduce the mathematical concept. Erratum: the limits of the Convolution Integral for the bixel are not infinity and minus infinity. The limits are the values of u1' and uz' for the pixel of the wedge surface that correspond to the selected bixel. To carry out calculations for a complete Treatment Planning Optimization and Computational Software Development, it is necessary to also include the rotation angles of the gantry and the couch. This done by implementing the corresponding matrices of rotations, and taking into account the Isocentre Position. This matter goes beyond the scope of this Technical Paper, which is focused only on the wedges Pencil-Beam pathway. Note also that this Superposition-Convolution Integral Model corresponds to the initial stages of the AAA algorithm development in water (constant density). It is not too complicated to implement these calculations on the recent AAA Formulas fitted for heterogeneus tissues with the necessary correction factors.Erratum:in convolution formula $\mathrm{z}$ must be z'. 


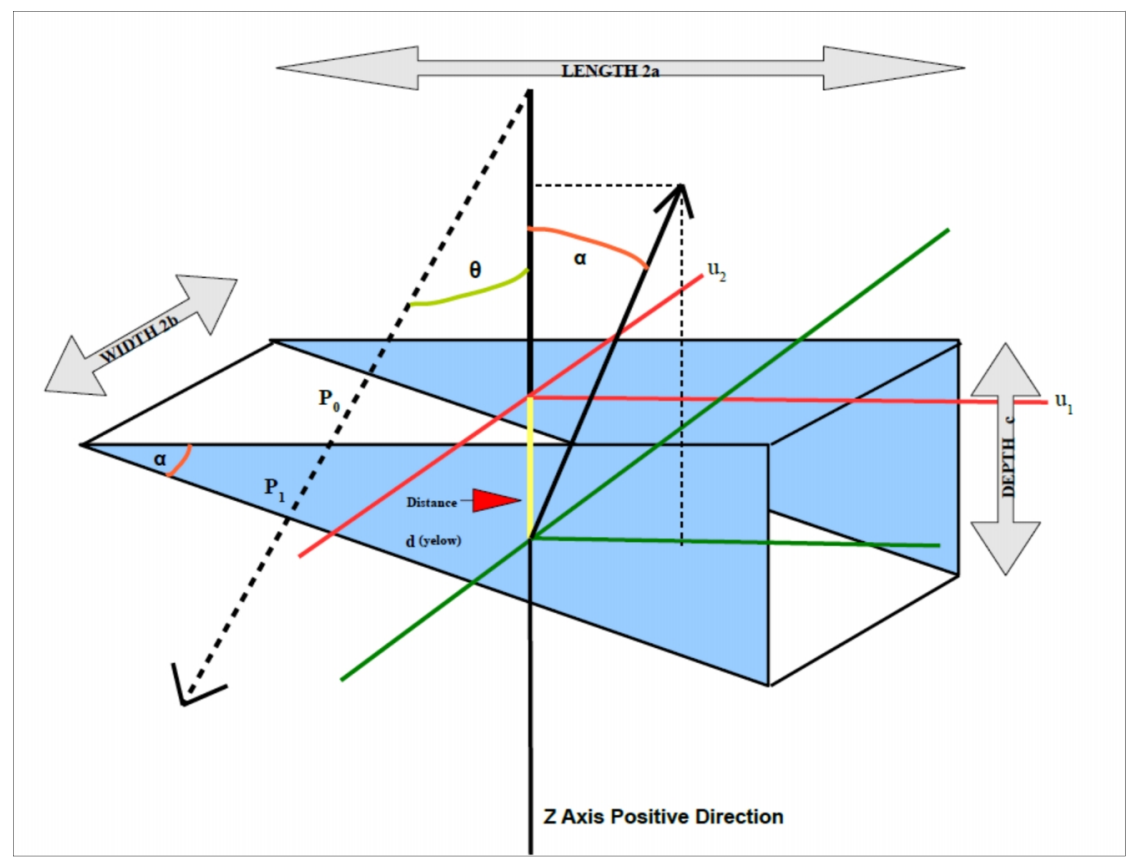

FIG. 3: The intent here is to provide an easy path to understanding of the Geometrical method. The unitary vector $\mathbf{n}$, which is perpendicular to the inferior plane, is sketched with its projections. An arbitrary Pencil-Beam is drawn on the left to show the input and output points upon the wedge volume. The broad part of the wedge corresponds to the positive quadrant of the superior $\mathrm{U}$ plane. Simulations were carried out just in this part, at the positive quadrant.

Note that the positive Z-axis is towards the Isocenter and patient surface. In the articles of foundation of the AAA Model, the measurements of the wedge are taken similar to the pictured one. That is, $\mathbf{2 a}, \mathbf{2 b}$, and $\mathbf{2 c}$ (we choose $\mathrm{c}$ instead $2 \mathrm{c}$ for simplicity).

We remind that a vector in 3D Analytic Geometry is defined by the difference of two points. The directional point (arrow) and the origin point (usually at coordinate center). In the calculations the origin of the perpendicular vector is taken at coordinate centre, but in the picture is translated at the inferior plane surface to put forward the problem geometry with clarity.

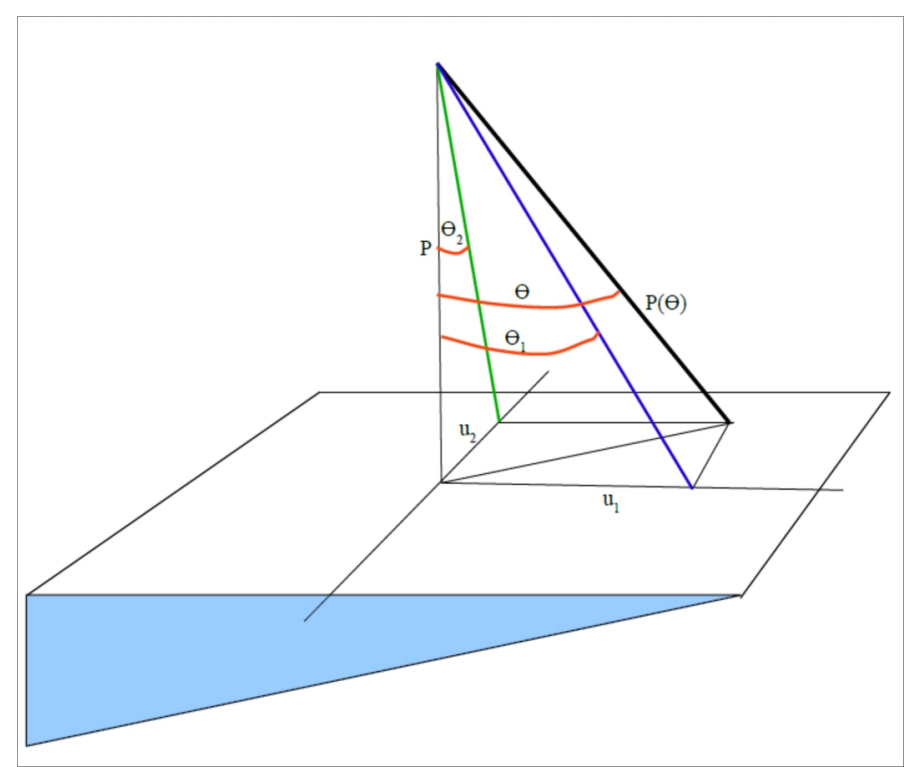

FIG. 4: We show the Pencil-Beam Divergence Angle subdivision to get smaller angles in order to better approximate the tangent value by Taylor Series, to the angle value in radians (first term of series, more options with more terms could be useful, but these are not linear). Note that this positive quadrant is put at the broad part of the wedge for simulations. 
All the parameters are tabulated. ${ }^{8-11}$ The derivation of the coefficient $\mathrm{ck}$ with the help of the Fourier transform, have already been described in the papers. ${ }^{8-9}$ Tabulations of I(z), $\sigma_{\mathrm{k}}(\mathrm{z})$, and $\mathrm{ck}$, based on Monte Carlo calculations of photon pencil beams for Co- 60 gamma radiation and bremsstrahlung from 6 to $18 \mathrm{MV}$, have already been published. ${ }^{8-9}$ This triple-Gaussian representation of the pencil beam has been chosen because its convolution with the photon flux distribution $\Phi(\mathrm{x}, \mathrm{y}, \mathrm{z})$ at depth $\mathrm{z}$ can be analytically performed in many practical cases. An important contribution to the savof computer time and storage space is thereby achieved, because numerical convolutions or applications of look-up tables from their fitting formulas are partially avoided. The analytical form of the resulting dose distributions may also offer other, yet unknown, applications. The triple-Gaussian pencil beam approach can be applied to radiation beam profiles that represent rectangular satellite blocks and wedge filters, as it is the case of this paper. The derivation of the coefficients ck with the help of the Fourier transform, have already been described in the papers. ${ }^{9-11}$ The term 'Superposition' comes from the sum of three Gaussians into the integral. The term 'Convolution' comes from the mathematical transformation carried out into the Dose-Deposition Kernel at the Integral.

With this Triple Gaussian $\mathrm{D}_{\mathrm{p}}(\mathrm{r}, \mathrm{z})$, a Kernel $\mathrm{K}(\mathrm{x}, \mathrm{y}, \mathrm{u}, \mathrm{v})$ was constructed to implement the dose term into the integral expression for the initial Superposition-Convolution Model in water, and then, the integral dose results in general as follows,

$D(x, y, z)=\int_{-\infty}^{\infty} \int_{-\infty}^{\infty} I(z) \Phi(x, y, z) K(x, y, z) d s----(3)$

where $\mathrm{I}(\mathrm{z})$ is the area integral of the absorbed dose over a plane perpendicular to the pencil beam axis at depth $\mathrm{z}$ per incident photon [33], $\Phi$ is the photon flux distribution of the beam per unit of intensity, and $\mathrm{K}$ is the kernel expression corresponding to the $\mathrm{PBM}$, which is called the $\mathrm{PB}$ dose kernel, and describes the spatial distribution of the absorbed energy. This kernel could perfectly include any other PBM, for example the classic Anesjö model ${ }^{12}$ or others. Now we focus on the aim of this research. The complete Triple Gaussian Pencil beam Model for water, when using wedges angle $\alpha$ and taking into account the Collimator Divergence Angle (usually very small), $\theta$ then reads (see Figure 2),

$\left.\begin{array}{l}D\left(x_{1}^{\prime}, x_{2}^{\prime}, z^{\prime}\right)=I\left(\alpha, z^{\prime}\right) \int_{-2 a}^{2 a} \int_{-2 b}^{2 b} \Phi_{z^{\prime}}\left(\alpha, \theta, u_{1}^{\prime}, u_{2}^{\prime}, z^{\prime}\right) \times \\ \sum_{k=1}^{3} \frac{c_{k}}{\pi \sigma_{k}^{2}\left(z^{\prime}\right)} e^{-\left(\left(x_{1}^{\prime}-u_{1}^{\prime}\right)^{2}+\left(x_{2}^{\prime}-u_{2}^{\prime}\right)^{2}\right) / \sigma_{k}^{2}\left(z^{\prime}\right)} d u_{1}^{\prime} d u_{2}^{\prime}\end{array}\right\}---(4)$

Eq (4) [erratum, limits of integral -a, a, -b, b]

The Flux (and the Intensity) change with an exponential attenuation factor that depends on the alloy material, $\mu_{\mathrm{w}}$, and the pathlength through the wedge $\mathrm{Lx}_{\mathrm{k}}$ in this way,
$\Phi_{z^{\prime}}\left(\alpha, \theta, u_{1}^{\prime}, u_{2}^{\prime}, z^{\prime}\right)=\Phi_{i}\left(u_{1}^{\prime}, u_{2}^{\prime}, z^{\prime}\right) \times e^{-\mu_{w} L_{K}\left(\alpha, \theta, u_{1}^{\prime}, u_{2}^{\prime}, z^{\prime}\right)} \ldots--(5)$ (see Footnote 1)

This point will be explained in detail in the next subsection. The intention here is to show the complete formula used to obtain the geometrical results, as we are only interested in determination of Lk. Recently ${ }^{9}$, a new contribution by the same authors for a 3D superposition-convolution algorithm based on their already developed, Triple Gaussian Pencil Beam Model (TGPBM). This algorithm has been built up results of Monte Carlo calculations. This superposition-convolution algorithm sorts the density changes of the tissue heterogeneities. An essential result of this algorithm is the determination of build-up and build-down effects in the domain of the density discontinuities. The corresponding practical measurements show a standard deviation of about $2 \%$. We won't describe the long theoretical framework and development of this algorithm, because what is of practical importance for the understanding is that the complete dose distribution formula (41) of the article ${ }^{10-11}$ for heterogeneous tissues reads,

$$
\begin{aligned}
& D(x, y, z)=\sum_{\beta}\left(1+z_{\beta} / S A D_{\beta}\right)^{-2}\left(\Phi_{\beta}\left(x_{\beta}, y_{\beta}^{\prime}, z_{\beta}\right) \times\right. \\
& \times \iint\left(H_{\beta}\left(u_{\beta}-x_{\beta}, v_{\beta}-y_{\beta}, z_{\beta}^{\prime}\right) I_{\beta}\left(z_{\beta}^{\prime}, \rho\left(z_{\beta}^{\prime}\right)\right)-\right. \\
& -\left(E_{\text {el }, \beta}\left(z_{\beta}, \rho\left(z_{\beta}\right)\right)-\Phi_{\text {cont }, \beta}\left(z_{\beta}, \rho\left(z_{\beta}\right)\right)\right) \times \\
& \left.\times K_{\text {el }, \beta}\left(u_{\beta}-x_{\beta}, v_{\beta}-y_{\beta}, z_{\beta}\right)\right) \times d u_{\beta} d v_{\beta}+ \\
& \left.+E_{\text {release }}\left(x_{\beta}, y_{\beta}, z_{\beta}\right)\right)
\end{aligned}
$$

Where $\beta$ is the number of pencil beams, $\mathrm{SAD}_{\beta}$ is the focus to surface distance of the pencil $\beta, \Phi_{\beta}$ is the flux for the $\beta$ pencil beam, $\mathrm{H}_{\beta}$ is the kernel containing the pencil beam model (we can choose the model (that is, $\left.\mathrm{H}\left(\mathrm{u}-\mathrm{x}^{\prime}, \mathrm{v}-\mathrm{y}^{\prime}, \mathrm{z}^{\prime}\right)\right)$. $\mathrm{I}_{\beta}$ is the area integral of the absorbed dose and its expression is given in formula (33) of the Ulmer et al articles ${ }^{8-11}$, Eel, $\beta$ is the energy of the electrons in the $\beta$ pencil beam given again by formula (22), $\Phi_{\mathrm{el}, \mathrm{cont}}$ is the flux of the contamination particles, mainly electrons, and given by formula (33), $\mathrm{Kel}_{\mathrm{e}, \beta}$ is the dose deposition kernel due to contamination electrons and is adapted by formula (23). Finally, Erelease is the energy released by local disequilibrium of forward and backward scatter and given by the formula (38). To implement in software and use this formula in mathematical calculations may appear to be difficult, but several parts have a simple development and others result null in specific cases.

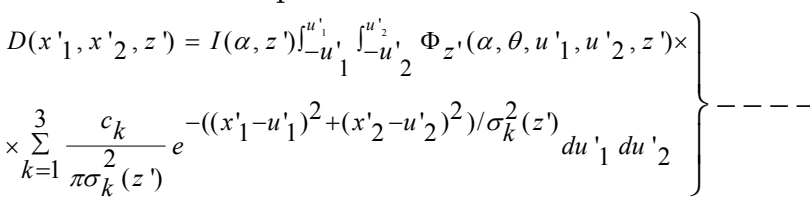

Where $\Phi_{z^{\prime}}\left(\alpha, \theta, u^{\prime} 1, u^{\prime} 2, z\right)$ is of the form (depending on the wedge angle alpha and the divergence angle theta): 
$\left.\begin{array}{l}\Phi_{z^{\prime}}\left(\alpha, \theta, u_{1}^{\prime}, u_{2}^{\prime}, z^{\prime}\right)=\Phi_{i}\left(u_{1}^{\prime}, u_{2}^{\prime}, z^{\prime}\right) \times e^{-\mu_{w} L_{K}\left(\alpha, \theta, u_{1}^{\prime}, u_{2}, z^{\prime}\right)} \\ \Phi_{i}\left(u_{1}^{\prime}, u_{2}^{\prime}, z^{\prime}\right)=\frac{\Phi_{w}}{\left[P(\theta)+z^{\prime}\right]^{2}}\end{array}\right\}---(8,9)$

Because of we apply the Inverse Square Law (ISL) of flux propagation for photons. ${ }^{1}$ That is, we take as reference the wedge surface flux for $\Phi_{\mathrm{W}}$ and this value decreases along the coordinate of the pencil beam according to the ISL. In the same way, the variation of the coordinates values from the pixel of wedge filter surface to the bixel base can be determined by trigonometric proportions from the triangles of the quasi-differential pyramid of the bixel as follows,

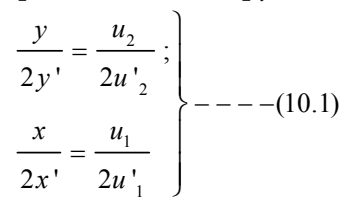

$\Phi_{\mathrm{W}}$ is the uniform initial flux incident on the upper wedge plane $U$. And $\mu_{\mathrm{w}}$ is the attenuation coefficient of the wedge material and $\mathrm{LK}_{\mathrm{K}}=\mathrm{LK}\left(\alpha, \theta, \mathrm{u}^{\prime} 1, \mathrm{u}^{\prime} 2, \mathrm{z}^{\prime}\right)$ is the distance,

$D=\left\|P_{1}-P_{0}\right\|=L_{K}-\cdots(10.2)$

that the pencil beam $\mathrm{P}(\Theta)$ travels into the wedge. The quantity $\mathrm{I}\left(\alpha, \mathrm{z}^{\prime}\right)$ can be approximately calculated from the empirical formula,

$\left.\begin{array}{l}I(\alpha, z)=\left(d_{1}+d_{2}\left(\mu^{\prime}(\alpha) z\right)^{q}\right) e^{-\mu^{\prime}(\alpha) z} \\ \text { where } \mu^{\prime}(\alpha)=\mu\left(1-C_{w} \tan \alpha\right)\end{array}\right\}----(11)$

However, the calculation of Intensity related to wedge angle is not the focus of the paper. These formulas are well detailed in the references about the primary stages of the AAA model. The parameters $\mathrm{d}_{1}, \mathrm{~d}_{2}, \mu, \mathrm{q}$, and $\mathrm{C}_{\mathrm{w}}$ are tabulated. ${ }^{9,10}$

The modification is required because the wedge changes the

\section{1}

We detail the mathematical development of the ISL applied on Flux calculations; we have at collimation output point a flux, $\Phi_{0}$, then, at wedge surface (still air) we get (ISL) as detailed in [Eq 9], then and at any point z' of the bixel, for the same ISL,

$$
\begin{aligned}
& \Phi_{z^{\prime}}=\frac{\Phi_{0}}{\left[P(\theta)+z^{\prime}\right]^{2}} e^{-\mu_{w} L_{K}\left(\alpha, \theta, u_{1}^{\prime}, u_{2}^{\prime}, z^{\prime}\right)}= \\
& =\frac{\Phi_{w}[P(\theta)]^{2}}{\left[P(\theta)+z^{\prime}\right]^{2}} e^{-\mu_{w} L_{K}\left(\alpha, \theta, u_{1}^{\prime}, u_{2}^{\prime}, z^{\prime}\right)}
\end{aligned}
$$

which is the same expression of [Eqs 8, 9]. energy spectrum of the photon beam. The integral detailed above is only an approximation for the dose, since the patch of the patient surface is not necessarily a plane, which is oriented transversally to $\mathrm{Z}^{\prime}$ axis.

$$
\left.\begin{array}{l}
D\left(x_{1}^{\prime}, x_{2}^{\prime}, z^{\prime}\right)=\frac{I\left(\alpha, z^{\prime}\right)}{\left[\frac{P(\theta)+z^{\prime}}{P(\theta)}\right]^{2}} \int_{-u_{1}^{\prime} u_{1}^{\prime}}^{u_{2}^{\prime} u_{2}^{\prime}} \times \Phi_{i}\left(u_{1}^{\prime}, u_{2}^{\prime}, z^{\prime}\right) \times \\
\times e^{-\mu_{w} L_{K}\left(\alpha, \theta, u_{1}^{\prime}, u_{2}^{\prime}, z^{\prime}\right)} \times \\
\times \sum_{k=1}^{3} \frac{{ }_{k}}{\pi \sigma_{k}^{2}\left(z^{\prime}\right)} e^{-\left(\left(x_{1}^{\prime}-u_{1}^{\prime}\right)^{2}+\left(x_{2}^{\prime}-u_{2}^{\prime}\right)^{2}\right) / \sigma_{k}^{2}\left(z^{\prime}\right)} d u_{1}^{\prime} d u_{2}^{\prime}
\end{array}\right\}
$$

In this Formula we have set the limits for the Coordinates $\mathrm{u}_{1}$ and $\mathrm{u}_{2}$ related to the difference of vectors $\mathrm{u}_{1}$ and $\mathrm{u}_{2}$ that dethe Coordinates System of the Central Axis Beam. There are other techniques to sort these calculation(s). Here Lk ( $\alpha, \theta$, $\left.\mathrm{u}^{\prime} 2, \mathrm{z}^{\prime}\right)$ is the distance $\mathrm{D}$ of the pathway through the wedge. This integral is developed numerically (Gauss-Quadrature preferably) to obtain the dose for this bixel. To determine total dose-deposition, it is necessary to add another summation which gathers all the bixels which comprise totally the wedge surface, and therefore the summation of the Pencil-Beams (each one with proper characteristics as it is done in IMRT). ${ }^{13-16}$

\section{Geometrical calculations}

In this Section we show the Analytic Geometry calculations to obtain the exact length of the pathway of a pencil beam when it goes through a point of the wedge upper, (superior) surface, $\mathrm{P}_{0}$, and emerges in air from the lower, (inferior) surface of the wedge at $\mathrm{P}_{1}$ [Figure 3 ]. This distance $\mathrm{D}$, is given in $3 \mathrm{D}$ by the formula,

$$
D=\left\|P_{1}-P_{0}\right\|=L_{K}-\cdots
$$

The method designed to find the formula for the Distance D, has the following steps,

I. Determination of the 3D Equations of the superior and inferior plane (at an alpha angle with the superior plane).

II. Find the 3D straight line equation (the Pencil Beam quasi-differential cylinder of very small radius) that goes from the collimator output window to the point $\mathrm{P}_{0}$ on the wedge surface.

III. Find the intersection point between the inferior plane of the wedge and this line. That is, the point $\mathrm{P}_{1}$.

IV. Calculate the distance D with the above formula.

V. The Distance D mathematical formula is simplified and formatted correctly to carry out convenient approximations (next section Approximations and mathematical analysis).

In Figure 2 we show the basic calculation system of the distance $\mathrm{D}$, and the corresponding Coordinate System. The wedge superior plane, since the main coordinates system is 
the center of the superior surface is,

$z=0----(14)$

To obtain the inferior plane equation we use the vectorial formula for a plane equation in $3 \mathrm{D}$, with a dot product,

$\vec{n} \bullet\left(\vec{x}-\vec{x}_{0}\right)=0----(15)$

That is, the coordinate system is ( $\left.\mathrm{u}_{1}, \mathrm{u}_{2}, \mathrm{z}\right)$, with coordinate origin in the superior plane of the wedge, and $\mathrm{Z}$ direction towards the beam propagation (that is, towards the patient body). We use a unitary vector of modulus $1, \mathrm{n}$, and component u2 null.

Since this vector is perpendicular to the lower wedge plane, it holds an angle alpha with $\mathrm{Z}$ Axis, and $\pi / 2-\alpha$ with the $\mathrm{u}_{1}$ axis. The wedge dimensions are $2 \mathrm{a}$ length, $2 \mathrm{~b}$ width, and $\mathrm{c}$ depth. Therefore, the maximum depth, $d$ ( $\mathrm{z}$ value) at the center is $\mathrm{d}=$ a $\tan \alpha$; [Figure 3]

The vector group corresponding to the above [see Figure 2] formula is [Eq 16]

$$
\left.\begin{array}{l}
\vec{n}=(\sin \alpha, 0,-\cos \alpha)^{T} ; \\
\vec{x}=\left(u_{1}, u_{2}, z\right)^{T} ; \\
\vec{x}_{0}=(0,0, a \tan \alpha)^{T} ;
\end{array}\right\}
$$

This yields, applying the previous equation with these vectors $\sin \alpha u_{1}-\cos \alpha z+a \sin \alpha=0----(17)$

which is the equation for the inferior plane. Next, we calculate the equation of the straight line that gives the trajectory of a pencil beam emerging from the collimator output with a small divergence angle $\theta$, and we apply the 3D Analytic Geometry Equation for the straight line,

$$
\begin{aligned}
& \left.\frac{x-x_{0}}{x_{1}-x_{0}}=\frac{y-y_{0}}{y_{1}-y_{0}}=\frac{z-z_{0}}{z_{1}-z_{0}}\right\}----(18 \\
& \text { where } x_{0}=\left(\begin{array}{c}
0 \\
0 \\
-P
\end{array}\right) ; \text { and } x_{1}=\left(\begin{array}{c}
u_{1} \\
u_{2} \\
0
\end{array}\right)
\end{aligned}
$$

We have two points, first, the output point, $\mathrm{X}_{0}$ of the collimator, as detailed above. Second, an arbitrary point at the superior wedge surface, with the coordinate system of the superior wedge surface, that is $\mathrm{X}_{1}$ as detailed above. Therefore, we get the straight line equations,

$$
\left.\frac{x}{u_{1}}=\frac{y}{u_{2}} ; \frac{y}{u_{2}}=\frac{z}{P}\right\}---
$$

And we have the third equation for the inferior plane [Eq 18], so we have a linear system of 3 equations in 3 variables, $\sin \alpha \mathrm{u}_{1}-\cos \alpha \mathrm{z}+\mathrm{a} \sin \alpha=0$;

The solution of the linear equations system is straightforward as follows,

$$
\begin{aligned}
& X_{\text {intersection }}=\frac{\frac{u_{1} a}{P}(-\sin \alpha)}{\frac{u_{1}}{P} \sin \alpha-\cos \alpha} \\
& Y_{\text {intersection }}=\frac{\frac{u_{2} a}{P}(-\sin \alpha)}{\frac{u_{1}}{P} \sin \alpha-\cos \alpha} ; \quad----(20) \\
& Z_{\text {intersection }}=\frac{a(-\sin \alpha)}{\frac{u_{1}}{P} \sin \alpha-\cos \alpha} ;
\end{aligned}
$$

The final step is to determine the distance,

$$
D=\left\|P_{1}-P_{0}\right\|=L_{K}-\cdots(21)
$$

Whose expression is,

$$
\left.D^{2}=\operatorname{Arg}\left\|\left(\begin{array}{c}
u_{1} \\
u_{2} \\
0
\end{array}\right)-\left(\begin{array}{c}
x_{\text {int } e r \text { section }} \\
y_{\text {int } \operatorname{rsection}} \\
z_{\text {int } r \text { section }}
\end{array}\right)\right\|^{2}=L_{K}^{2}\right\}----(22)
$$

With the result,

$$
\begin{aligned}
& D^{2}=\left[u_{1}-\frac{\frac{u_{1} a}{P}(-\sin \alpha)}{\frac{u_{1}}{P} \sin \alpha-\cos \alpha}\right]^{2}+ \\
& +\left[u_{2}-\frac{\frac{u_{2} a}{P}(-\sin \alpha)}{\frac{u_{1}}{P} \sin \alpha-\cos \alpha}\right]^{2}+\left[\frac{a(-\sin \alpha)}{\frac{u_{1}}{P} \sin \alpha-\cos \alpha}\right]^{2}
\end{aligned}
$$

According to Figures 2, 3, and 4, we can introduce in the above formula the collimator pencil-beam divergence angle as follows, $P=P(\theta) \cos \theta$. This substitution works if we want to implement the $\theta$ variable in the general formula. In the next section we detail the approximations to make this rather complicated formula easier to calculate and simplify it as much as possible. According to the exponential attenuation factor into the integral dose formula $\mathrm{D}=\mathrm{Lk}$ (Exact Pencil-Beam Pathway Value). This substitution works if we want to implement the $\theta$ variable in the general formula. In the next section we detail the approximations to make this rather complicated formula easier to calculate and simplify it as much as possible. According to the exponential attenuation factor into the integral dose formula D $=$ Lk (Exact Pencil-Beam Pathway Value).

\section{Approximations and mathematical analysis}


We start with the previous rather extensive formulation of [Eq 23],

$$
\begin{aligned}
& D^{2}=\left[u_{1}-\frac{\frac{u_{1} a}{P}(-\sin \alpha)}{\frac{u_{1}}{P} \sin \alpha-\cos \alpha}\right]^{2}+ \\
& +\left[u_{2}-\frac{\frac{u_{2} a}{P}(-\sin \alpha)}{\frac{u_{1}}{P} \sin \alpha-\cos \alpha}\right]^{2}+\left[\frac{a(-\sin \alpha)}{\frac{u_{1}}{P} \sin \alpha-\cos \alpha}\right]^{2} ;
\end{aligned}
$$

Which was set previously as, $\mathrm{P}=\mathrm{P}(\theta) \cos \theta$.

Now, we will define two Pencil-Beam Divergence Angles related to $\theta$, namely, $\theta_{1}$ and $\theta_{2}$ with the trigonometric conditions of Figures 4 , and, $\tan \theta_{1}=\mathrm{u}_{1} / \mathrm{P}$ and $\tan \theta_{2}=\mathrm{u}_{2} / \mathrm{P}$.

\section{Proposition 1}

In pencil-beam angle decomposition, $\theta_{1}$ and $\theta_{2}$ are always less than or equal to $\theta$.

The objective of this Proposition is to carry out approximations with the smallest angle values possible, making series developments optimal.

\section{Proof}

Since (Figure 4),

$\left.\begin{array}{l}u_{1} \leq \sqrt{u_{1}^{2}+u_{2}^{2}} \\ u_{2} \leq \sqrt{u_{1}^{2}+u_{2}^{2}}\end{array}\right\}----(2$

then, $\tan (\theta)=\frac{\sqrt{u_{1}^{2}+u_{2}^{2}}}{p}$;

and, $\left.\tan \left(\theta_{1}\right)=\frac{u_{1}}{p} \leq \tan (\theta) ;\right\}$

with, $\tan \left(\theta_{2}\right)=\frac{u_{2}}{p} \leq \tan (\theta) ;$

Therefore, the Proposition is proven.

And, considering series development for tangent small angles, the following conditions will be accomplished,

$\left.\begin{array}{l}\theta_{1} \leq \theta_{2} \\ \theta_{2} \leq \theta_{2}\end{array}\right\}----(26)$

which was the objective to minimize errors in the approximations. Then, we carry out the following changes to inthese divergence angle(s) and simplify the initial formula (Exact Pencil-Beam Pathway Formula),

$$
\left.\begin{array}{l}
D^{2}=\left[u_{1}-\frac{\tan \theta_{1} a(-\sin \alpha)}{\tan \theta_{1} \sin \alpha-\cos \alpha}\right]^{2}+ \\
+\left[u_{2}-\frac{\tan \theta_{2} a(-\sin \alpha)}{\tan \theta_{1} \sin \alpha-\cos \alpha}\right]^{2}+\left[\frac{a(-\sin \alpha)}{\tan \theta_{1} \sin \alpha-\cos \alpha}\right]^{2}
\end{array}\right\}
$$

Hence, making the previous trigonometric changes for small angles (Approximated Pencil-Beam Pathway Formula),

$$
\left.\begin{array}{l}
D^{2} \cong\left[u_{1}-\frac{\theta_{1} a(-\sin \alpha)}{\theta_{1} \sin \alpha-\cos \alpha}\right]^{2}+ \\
+\left[u_{2}-\frac{\theta_{2} a(-\sin \alpha)}{\theta_{1} \sin \alpha-\cos \alpha}\right]^{2}+\left[\frac{a(-\sin \alpha)}{\theta_{1} \sin \alpha-\cos \alpha}\right]^{2}
\end{array}\right\}----(28)
$$

Now we have only 5 linear variables within the brackets of the formula, namely, $\mathrm{u}_{1}, \mathrm{u}_{2}, \theta_{1}, \theta_{2}, \theta$ (optional) and the wedge angles constant $\alpha$.

\section{Computational simulations and software}

A series of simple and basic simulations [Annex A] were carried out to check the accuracy of the approximations and the algebraic development. Simulations were made at the positive quadrant of the thick part of the wedges.The accuracy is given by the error formula of section Error calculations \& further approximations. The software used was FreeMat v4.0 (Copyright (c) 2002-2008 by Samit Basu Licensed under the GNU Public License (GPL)) programming and Optimization Subroutines both in single and double precision [C4]. The trajectory of the Pencil Beam for each simulation and the corresponding divergence angle were selected randomly.

The divergence angle interval was chosen narrow, since the divergence angles are usually small in modern accelerators which work with IMRT. It is very important to clarify that when the wedge angle increases, the simulation quadrant for $\mathrm{u}_{1}$ and $\mathrm{u}_{2}$ has to be reduced. The geometrical reason is that there is a Limit Divergence Angle $\theta$ for the pencil beam. If this divergence angle is surpassed, the outpoint of the beam after passing through the wedge is located at the lateral side/wall of the wedge (we refer mainly to the broad part of the wide-angle wedge). This inconvenient is especially present when we simulate paths through wide-angle wedges. In Annex A, some details about the Limit Angle are specified, mainly concerning wedges with wide angle, ie, 45을 60 .

\section{Error calculations \& further approximations}

The calculation of the Error for a wedge of angle alpha is the Standard RMS Equation where we measure the average quadratic difference between the exact pathway along the wedge material and the approximated path, according to the calculations carried out in section Approximations and mathematical analysis. The RMS Error Formula reads, 


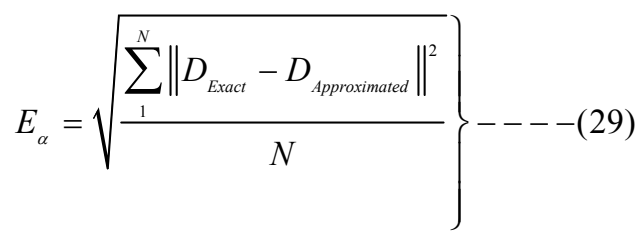

Where $\mathrm{N}$ is the number of random pathways selected in the simulations. Note that in Annex A Tables, we write positive error values, but negative are also mathematically considered.

The 10 simulations carried out for, i.e. a $30^{\circ}$ Wedge give a RMS Error [Table 2], in the order of $10^{-3}$. The tangent approximation in series development results in small errors because the angles at $\mathrm{P}=20 \mathrm{~cm}$ (distance from the Collimator Output) are also small. The random numbers interval chosen was at the positive quadrant of the broad zone. Usually, the approximated values are larger than the exact ones.

For mathematical analysis, it is both practical and useful to determine what we define as Exponential RMS Error. That when we use approximations, the exponential attenuation factor of the wedge filter is set into an interval determined the numerical errors. Specifically, we are calculating an approximated Lk parameter, which is in the exponent of an Integral Exponential Factor (IEF). We have, without approximations,

Integral Exponential Factor $(I E F) \cong e^{-\mu_{w} L_{K}\left(\alpha, \theta, u^{\prime}, u^{\prime}, z^{2}\right)}$

Then, since we have determined an approximated Lk with RMS Error, the True L' $\mathrm{k}$ will be determined within the error interval, so we set a standard Confidence Interval as,

$L_{K}^{\prime} \in\left[L_{K}-3 \varepsilon, L_{K}+3 \varepsilon\right]$

and the modified True Integral Exponential Factor reads,

True Exponential Factor $(T E F) \cong$

$\cong e^{-\mu_{w} L_{K}^{\prime}\left(\alpha, \theta, u_{1}^{\prime}, u_{2}^{\prime}, z^{\prime}\right)} \in$

$\left[e^{-\mu_{w}\left[L_{K} \pm 3 \varepsilon\right]\left(\alpha, \theta, u_{1}^{\prime}, u_{2}^{\prime}, z^{\prime}\right)}\right]=$

$=\left[e^{-\mu_{w} L_{K}\left(\alpha, \theta, u_{1}^{\prime}, u_{2}^{\prime}, z^{\prime}\right)} \times\right.$

$\left.\times e^{-\mu_{w}[ \pm 3 \varepsilon]\left(\alpha, \theta, u_{1}^{\prime}, u_{2}^{\prime}, z^{\prime}\right)}\right]=$

$=\left[I E F \times e^{-\mu_{w}[+3 \varepsilon]\left(\alpha, \theta, u_{1}^{\prime}, u_{2}^{\prime}, z^{\prime}\right)}\right.$,

,IEF $\left.\times e^{-\mu_{w}[-3 \varepsilon]\left(\alpha, \theta, u_{1}^{\prime}, u_{2}^{\prime}, z^{\prime}\right)}\right]$
In consequence, we define the RMS Error Integral Exponential Factor, to set a trustworthy Confidence Interval (Note that the width of the Confidence Interval can be chosen larger or smaller), as follows,

Error Integral Exponential Factor $(\mathrm{EIEF}) \cong e^{-\mu_{w}[ \pm 3 \varepsilon]\left(\alpha, \theta, u_{1}^{\prime}, u_{2}^{\prime}, z^{\prime}\right)}$

A numerical example with the obtained simulation data at 30 whose Absolute Error is 0.0441 (Annex A Table 2) is,

Error Integral Exponential Factor $($ EIEF $) \cong e^{-\mu_{w}[ \pm 3 \times 0.0441]\left(\alpha, \theta, u_{1}{ }_{1}, u_{2}^{\prime}, z^{\prime}\right)}=$ $=e^{-\mu_{w}[ \pm 0.1323]\left(\alpha, \theta, u_{1}^{\prime}, u_{2}^{\prime}, z^{\prime}\right)}$

And the Exact Dose Confidence Interval, taking the Average Lk value for $30 \circ(10.3197 \mathrm{~cm})$ is,

$$
\begin{aligned}
& D_{\text {Exact }} \in \\
& \in\left[D \text { for } e^{-\mu_{w}[10.3197 \pm 0.1323]\left(\alpha, \theta, u_{1}^{\prime}, u_{2}^{\prime}, z^{\prime}\right)}\right]= \\
& =\left[D \text { for } e^{-\mu_{w} \times 10.3197\left(\alpha, \theta, u_{1}^{\prime}, u_{2}^{\prime}, z^{\prime}\right)} \times\right. \\
& \left.\times e^{-\mu_{w}[ \pm 0.1323]\left(\alpha, \theta, u_{1}^{\prime}, u_{2}^{\prime}, z^{\prime}\right)}\right]= \\
& =\left[D \text { for IEF } \times e^{-\mu_{w}[+0.1323]\left(\alpha, \theta, u_{1}^{\prime}, u_{2}^{\prime}, z^{\prime}\right)},\right. \\
& \left., D \text { for IEF } \times e^{-\mu_{w}[-0.1323]\left(\alpha, \theta, u_{1}^{\prime}, u_{2}^{\prime}, z^{\prime}\right)}\right] ;
\end{aligned}
$$

With $\mu_{\mathrm{w}}$ values for Alloy tabulated [U2].

We also set the RMS Average Error Integral Exponential Factor, as the average of the distance D Error $\varepsilon \mathrm{A}$, after we have taken sufficient random RMS measurements along all the wedge surface. These measurements have to take into account the Limit Divergence Angle, $\theta_{\mathrm{L} .}$ Limit Angle mathematical details are explained in section Beam limit divergence angle (LA) concept and formulation with appropriate formulation. Then, this Integral factor reads,

Average Error Integral Exponential Factor $\cong e^{-\mu_{w}\left[ \pm 3 \varepsilon_{A}\right]\left(\alpha, \theta, u_{1}^{\prime}, u_{2}^{\prime}, z^{2}\right)}$

The exponential factors always can be approximated by sedevelopment. We do not carry out this kind of mathematical framework in this contribution, which will be developed in subsequent publications. The study of how much this kind of approximation of exponential is useful corresponds to approximation theory.

Hence, the Approximated Dose Integral, when using approximation LK (without Confidence Interval), will result, 


$$
\begin{aligned}
& D_{\text {Approximated }}\left(x_{1}^{\prime}, x_{2}^{\prime}, z^{\prime}\right) \cong I\left(\alpha, z^{\prime}\right) \times \int_{-2 a}^{2 a} \int_{-2 b}^{2 b} e^{-\mu_{w}\left[L_{K} \pm \varepsilon_{A}\right]\left(\alpha, \theta, u_{1}^{\prime}, u_{2}^{\prime}, z^{\prime}\right)} \times \\
& \times \Phi_{z^{\prime}}\left(\alpha, \theta, u_{1}^{\prime}, u_{2}^{\prime}, z^{\prime}\right) \times \sum_{k=1}^{3} \frac{c_{k}}{\pi \sigma_{k}^{2}\left(z^{\prime}\right)} e^{-\left(\left(x_{1}^{\prime}-u_{1}^{\prime}\right)^{2}+\left(x_{2}^{\prime}-u_{2}^{\prime}\right)^{2}\right) / \sigma_{k}^{2}\left(z^{\prime}\right)} \times \\
& \times d u_{1}^{\prime} d u_{2}^{\prime}=I\left(\alpha, z^{\prime}\right) \times \int_{-2 a}^{2 a} \int_{-2 b}^{2 b} e^{-\mu_{w}\left[L_{K}\right]\left(\alpha, \theta, u_{1}^{\prime}, u_{2}^{\prime}, z^{\prime}\right)} \times e^{-\mu_{w}\left[ \pm \varepsilon_{A}\right]\left(\alpha, \theta, u_{1}^{\prime}, u_{2}^{\prime}, z^{\prime}\right)} \times \\
& \times \Phi_{z^{\prime}}\left(\alpha, \theta, u_{1}^{\prime}, u_{2}^{\prime}, z^{\prime}\right) \times \sum_{k=1}^{3} \frac{c_{k}}{\pi \sigma_{k}^{2}\left(z^{\prime}\right)} e^{-\left(\left(x_{1}^{\prime}-u_{1}^{\prime}\right)^{2}+\left(x_{2}^{\prime}-u_{2}^{\prime}\right)^{2}\right) / \sigma_{k}^{2}\left(z^{\prime}\right)} \times d u_{1}^{\prime} d u_{2}^{\prime}=I\left(\alpha, z^{\prime}\right) \times \\
& \times e^{-\mu_{w}\left[ \pm \varepsilon_{A}\right]\left(\alpha, \theta, u_{1}^{\prime}, u_{2}^{\prime}, z^{\prime}\right)} \times \int_{-2 a}^{2 a} \int_{-2 b}^{2 b} e^{-\mu_{w}\left[L_{K}\right]\left(\alpha, \theta, u_{1}^{\prime}, u_{2}^{\prime}, z^{\prime}\right)} \times \Phi_{z^{\prime}}\left(\alpha, \theta, u_{1}^{\prime}, u_{2}^{\prime}, z^{\prime}\right) \times \\
& \times \sum_{k=1}^{3} \frac{c_{k}}{\pi \sigma_{k}^{2}\left(z^{\prime}\right)} e^{-\left(\left(x_{1}^{\prime}-u_{1}^{\prime}\right)^{2}+\left(x_{2}^{\prime}-u_{2}^{\prime}\right)^{2}\right) / \sigma_{k}^{2}\left(z^{\prime}\right)} \times d u_{1}^{\prime} d u_{2}^{\prime}
\end{aligned}
$$

And the Average Error Integral Factor has been set outside of the integrand because it is considered average and constant for all the values belonging to the integral Hence, the Approximated Dose Integral, when using approximation Lk (without Confidence Interval), will result,

$$
\left.\begin{array}{l}
D_{\text {Exact }} \in\left[D_{\text {Approx }} \text { for } e^{-\mu_{w}\left[+3 \varepsilon_{A}\right]\left(\alpha, \theta, u_{1}^{\prime}, u_{2}^{\prime}, z^{\prime}\right)},\right. \\
\left.D_{\text {Approx }} \text { for } e^{-\mu_{w}\left[-3 \varepsilon_{A}\right]\left(\alpha, \theta, u_{1}^{\prime}, u_{2}^{\prime}, z^{\prime}\right)}\right]
\end{array}\right\}----(31)
$$

A formula which is useful both for industrial manufacturing purposes or treatment planning optimization approximations when using static wedge filters. The Relative RMS Error Formula which has been applied in the tables of Annex A reads,

Relative RMS Error $= \pm\left[\frac{100 \times E_{\alpha}}{\bar{D}_{\text {Exact(Average) }}}\right]----$

where $E_{\alpha}$ is the RMS Absolute Error [Eq 29]. Note that in Annex A Tables we write positive error values, but negative are also mathematically considered. The Relative Approximation Error is under 5\% when the wedge angle is small, $15 \%$ or 30 , and rises about $10 \%$ when the angle is wider, i.e., $45 \circ$, or 60 . The explanation for this increment is that we use only the first term of the tangent series development. Therefore, when both the exact and approximated paths increase, the difference between them becomes higher for trigonometric reasons. A possible solution is to use more terms of the series, or develop further geometrical approximations.

Beam limit divergence angle (LA) concept and formulation In previous contributions 16,17 , the LA was mathematically defined and developed for wedges. We detail here the main formulas and one sketch of LA, together with a picture of the so-called Conformal Wedge. ${ }^{18}$ Given a fixed collimator to wedge surface distance, LA is defined as the maximum angle of divergence that can be reached by the whole radiation beam without emerging at any point of lateral of the wedge. Photon-Beam divergence angles values vary around 20 degrees. The Beam minimum divergence depends on the collimator design quality, and in general of the precision engineering manufacturing of the LINACs. LA is useful because of several reasons. Avoids hot spots, sub-optimal dose delivery, planning system software propagation errors, overdose at OARS, and repetition of planning work caused by sub-optimal dose delivery calculations. The LA for a conformal wedge calculation presents some additional difficulties. However, the primary approximation is to take as LA for a Conformal Wedge the value of the deepest step of the wedge. Main formulation for LA in standard wedges is for the principal pencil beam

$$
\theta_{\mathrm{L}}[\text { Geometrical }]=\operatorname{arctg}([\mathrm{r}] \mathrm{u} 2=0) / \mathrm{P}
$$

where

$r=b-\operatorname{tg} \theta \operatorname{tg} \alpha(b+a)=r(\theta)$

from [22]

$\mathrm{r}=\mathrm{b}-\operatorname{tg} \theta\left[\operatorname{tg} \alpha\left[\left(\mathrm{b}^{2}-\mathrm{u} 2^{2}\right)^{1 / 2}+\mathrm{a}\right]\right]=\mathrm{r}(\theta)$

where $\mathrm{P}$ is the distance between the collimator output and wedge surface (perpendicular, ${ }^{17}$ ) and we have used the constraint for inferior geodesic.$^{17}$

$\mathrm{u}_{1}^{2}+\mathrm{u}_{2}^{2}=\mathrm{b}^{2}$

Therefore, to make sure the components of the decomposed beam have a correct output point the following conditions should hold

$\left.\begin{array}{l}\theta_{1} \leq \arctan (\mathrm{a} /(\mathrm{P}+2 \mathrm{c}) \\ \theta_{2} \leq \arctan (\mathrm{a} /(\mathrm{P}+\mathrm{c}))\end{array}\right\}----(33)$

\section{Discussion and Conclusion}

The pathway in IMRT for static wedges has been determined using an integral Superposition-Convolution Model for Pencil Beam. The radiation delivery model selected was the 
Superposition-Convolution model with wedge attenuation factor.

The calculation result for 3D is according to the fundamenof Analytic Geometry. The applied Formulation is the standard in this kind of determinations. We have tried to clarify any kind of complicated mathematical development, formulation and expression. Additional approximations to avoid complicated functions, such as trigonometric or quadratic expressions, have been done taking into account the small divergence angle of the collimator output, $\theta$. These approximations were based mainly on Taylor series development related to trigonometric functions. For instance, the Numerical RMS Error for a $30^{\circ}$ Wedge series of 10 simulations is about $10^{-2}$ and this magnitude can be considered acceptable, present investigation stage. We found a Limit Divergence Angle $\theta$ related to the central beam, which constitutes an important parameter to avoid the output of the beam mainly through the lateral broad part of the wedge (this phenomenon happens mainly in wedge filters of wide angle). Details about this LA have been explained according to previous publications. Industrial applications of this method are focused on several RT areas. In IMRT, the use of verified theoretical data of the attenuation of the wedge its surface, could yield an improved clinical use of the IMRT distribution, and obtain a database for planning system improvements. In addition, it could be employed to develop better attenuation factors for RT mathematical modelling in IMRT or External Beam Radiotherapy (EBRT), according to the geometrical distribution data of the $3 \mathrm{D}$ paths along the wedge volume. Furthermore, Nonlinear Optimization Techniques could be used with Multiobjective Techniques ${ }^{19}$ for a better combination of the Attenuation Factor(s), wedge zone and IMRT Pencil Beam Intensity Attenuation Factor(s).

\section{Conflict of interest}

The authors declare that they have no conflicts of interest. The authors alone are responsible for the content and writing of the paper.

\section{References}

1. Capizzello A, Tsekeris PG, Pakos EE, et al. Adjuvant chemo-radiotherapy in patients with gastric cancer. Indian J Cancer 2006; 43:174-9.

2. Ragaz J, Olivotto IA, Spinelli JJ, et al. Locoregional radiation therapy in patients with high-risk breast cancer receiving adjuvant chemotherapy: 20-year results of the British Columbia randomized trial. $J$ Natl Cancer Inst 2005; 97:116-26.

3. Wilt TJ, Shamliyan T, Taylor B, et al. Comparative Effectiveness of Therapies for Clinically Localized Prostate Cancer [Internet]. AHRQ Comparative Effectiveness Reviews 2008.
4. Haas OC. Radiotherapy treatment planning: New systems approaches. Springer Engineering 1998.

5. Ulmer W, Schaffner B. Foundation of an analytical proton beamlet model for inclusion in a general proton dose calculation system. Radiation Physics and Chemistry 2011; 80: 378-402.

6. Numrich RW. The computational energy spectrum of a program as it executes. J Supercomput 2010; 52:119-34.

7. Sharma SC. Beam Modification Devices in Radiotherapy Lecture at Radiotherapy Department PGIMER, 2008.

8. Ulmer W, Harder D. A triple Gaussian pencil beam model for photon beam treatment planning, $\mathrm{Z}$. Med Phys 1995; 5: 25-30.

9. Ulmer W, Pyyry J, Kaissl W. A 3D photon superposition/convolution algorithm and its foundation on results of Monte Carlo calculations. Phys Med Biol2005; 50:1767-90.

10. Ulmer W. Laboratory Report. Phys Med Biol 50, 2005.

11. Ulmer W, Harder D. Applications of the triple Gaussian Photon Pencil Beam Model to irregular Fields, dynamical Collimators and circular Fields. Phys Med Biol 1997.

12. Ahnesjö A, Saxner M, Trepp A. A pencil beam model for photon dose calculations. Med Phys 1992; 19:263-73.

13. Hashemiparast SM, Fallahgoul H. Modified Gauss quadrature for ill-posed integral transform. International Journal of Mathematics and Computation. 2011; 13: D11.

14. Casesnoves F. Large-Scale Matlab Optimization Toolbox (MOT) Computing Methods in Radiotherapy Inverse Treatment Planning. High Performance Computing Meeting. Nottingham University. January 2007.

15. Casesnoves F. A Computational Radiotherapy Optimization Method for Inverse Planning with Static Wedges. High Performance Computing Conference. Nottingham University. January 2008.

16. Casesnoves F. Exact/Approximated Geometrical Determinations of IMRT Photon Pencil-Beam Path Through Alloy Static Wedges in Radiotherapy Using Anisothropic Analytic Algorithm (AAA)' peer-reviewed ASME Conference paper-poster. Proceedings of ASME 2011 IMECE (International Mechanical Engineering Conference) Conference. Denver, Nov 2011. CO.

17. Casesnoves F. Geometrical Determinations of Limit Angle (LA) related to Maximum Pencil-Beam Divergence Angle in Radiotherapy Wedges. ASME Conference Paper. ASME International Mechanical Engineering Congress. Houston, USA 2012.

18. Casesnoves F. A Conformal Radiotherapy Wedge Filter Design. Computational and Mathematical 
Model/Simulation. Peer-Reviewed Poster IEEE (Institute for Electrical and Electronics Engineers), Northeast Bioengineering Conference. Syracuse New York, USA. Presented in the Peer-Reviewed Poster Session on 6th April 2013. Sessions 1 and 3 with Poster Number 35. Page 15 of Conference Booklet.

19. Steuer R. Multiple Criteria Optimization: Theory, Computation and Application. Wiley 1986.

20. Kirsch A. An introduction to the Mathematical Theory of Inverse Problems. Spinger Applied Mathematical Sciences 1996.

21. Moczko JA, Roszak A. Application of Mathematical Modeling in Survival Time Prediction for Females with Advanced Cervical cancer treated Radio-chemotherapy. Computational Methods in science and Technology 2006; 12: 143-7.

22. Brahme A. Development of Radiation Therapy Optimization. Acta Oncol 2000; 39: 579-95.

23. Hong TS, Craft DL, Carlsson F, Bortfeld TR. Multicriteria optimization in intensity-modulated radiation therapy treatment planning for locally advanced cancer of the pancreatic head. Int J Radiat Oncol Biol Phys 2008; 72:1208-14.

24. Censor Y, Zenios SA. Parallel Optimization: Theory, Algorithms and Applications. UOP, 1997.

25. Casesnoves F. Determination of absorbed doses in common radiodiagnostic explorations. $5^{\text {th }}$ National Meeting of Medical Physics. Madrid, Spain. September 1985.

26. Censor Y. Mathematical Optimization for the Inverse problem of Intensity-Modulated Radiation Therapy. Laboratory Report, Department of Mathematics, University of Haifa, Israel, 2005.
27. Do SY, Bush DA, Slater JD. Comorbidity-adjusted survival in early stage lung cancer patients treated with hypofractionated proton therapy. J Oncol 2010; 2010:251208.

28. Ehrgott M, Burjony M. Radiation Therapy Planning by Multicriteria Optimization. Department of Engineering Science. University of Auckland. New Zealand.

29. Ezzel GA. Genetic and geometric optimization of three dimensional radiation therapy treatment planning. Med Phys 1996; 23: 293-305.

30. Hansen P. Rank-deficient and discrete ill-posed problems: Numerical aspects of linear inversion. SIAM monographs on mathematical modelling and computation 1998.

31. Johansson KA, Mattsson S, Brahme A, Carlsson J, Zackrisson B, Turesson I; Swedish Cancer Society Investigation Group. Radiation therapy dose delivery. Acta Oncol 2003; 42:85-91.

32. Kufer KH, Hamacher HW, Bortfeld T. A multicriteria optimisation approach for inverse radiotherapy planning. University of Kaiserslautern, Germany, Spring-Verlag Berlin Heidelberg 2000.

33. Luenberger DG. Linear and Nonlinear Programming $2^{\text {nd }}$ edition. Addison-Wesley, 1984.

34. Spirou SV, Chui CS. A gradient inverse planning algorithm with dose-volume constraints. Med Phys 1998; 25: 321-33.

35. Valachis A, Mauri D, Polyzos NP, et al. Partial breast irradiation or whole breast radiotherapy for early breast cancer: a meta-analysis of randomized controlled trials. Breast J2010; 16:245-51. 


\section{Annex A}

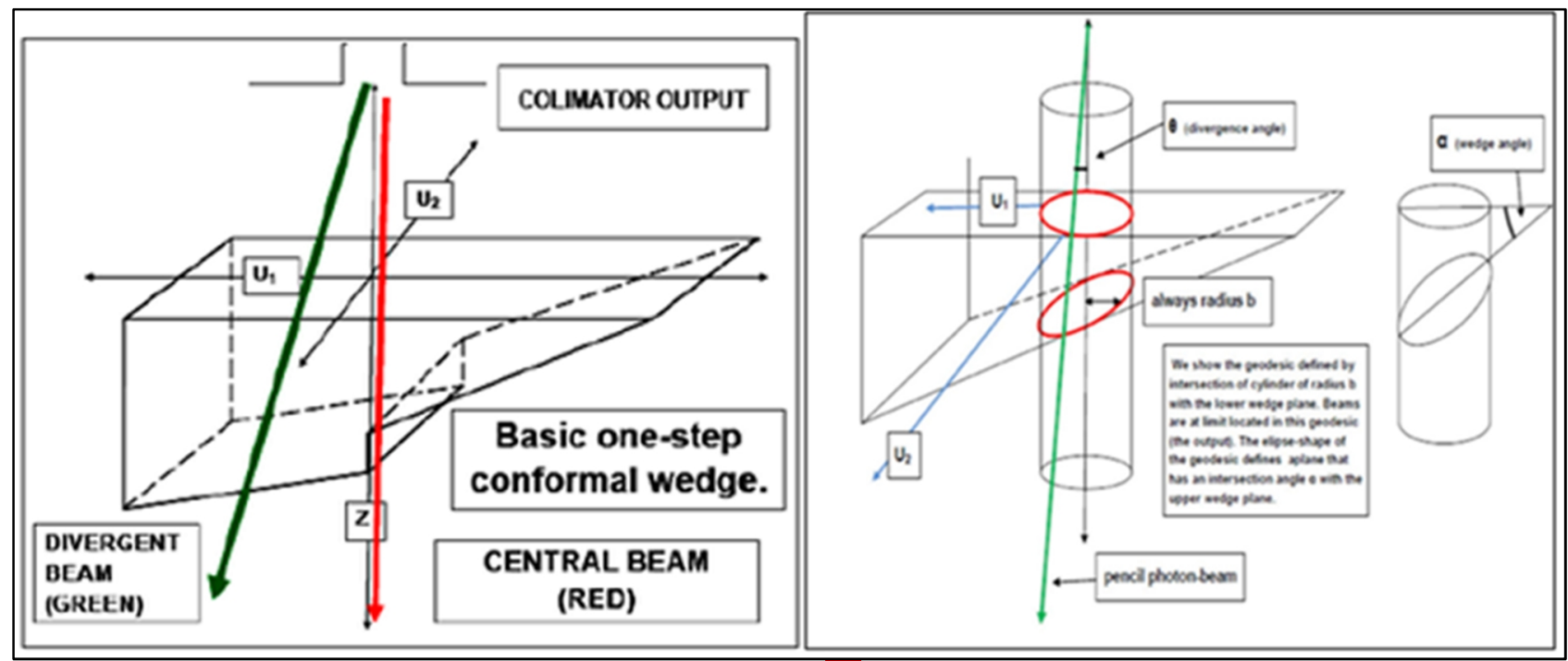

FIG. 5: Approximations for the LA in standard wedges ${ }^{17,18}$ and a picture of 2-steps conformal wedge. ${ }^{18}$

TABLE 1: $15^{0}$ Wedge filter angles. Simulation carried out at positive quadrant of the broad part of the wedge. Selected wedges are sloping surface straight type.

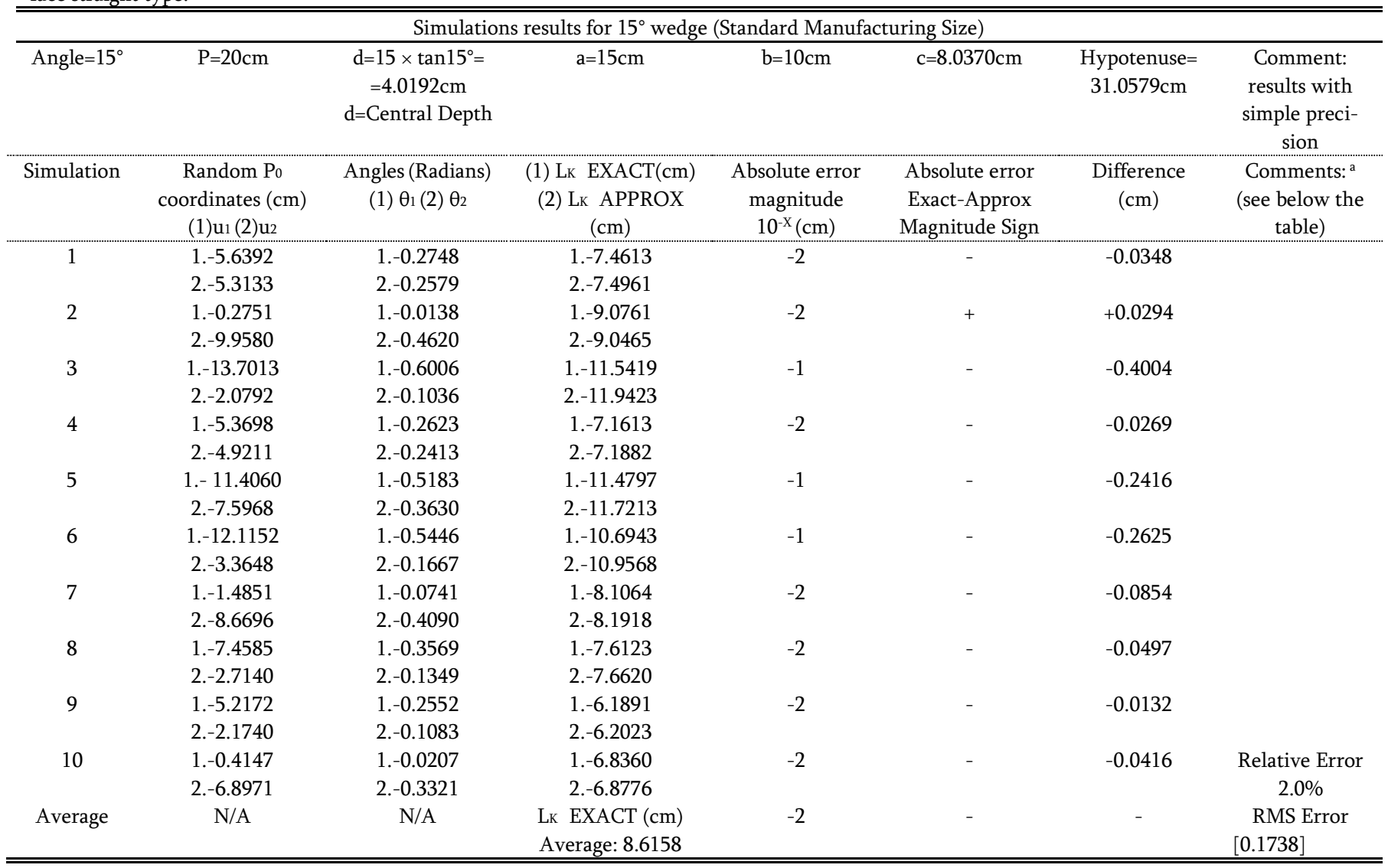

Comments: ${ }^{a}=$ Since the wedge angle is tight, the error values are low and the limit angle is not reached in the simulations. The chosen range of simulations is, $\mathrm{u}_{1} \in[0,15] \mathrm{u} 2 \epsilon[0,10]$ 
TABLE 2: $30^{\circ}$ Wedge filter angles. Simulation carried out at positive quadrant of the broad part of the wedge. Selected wedges are sloping surface straight type.

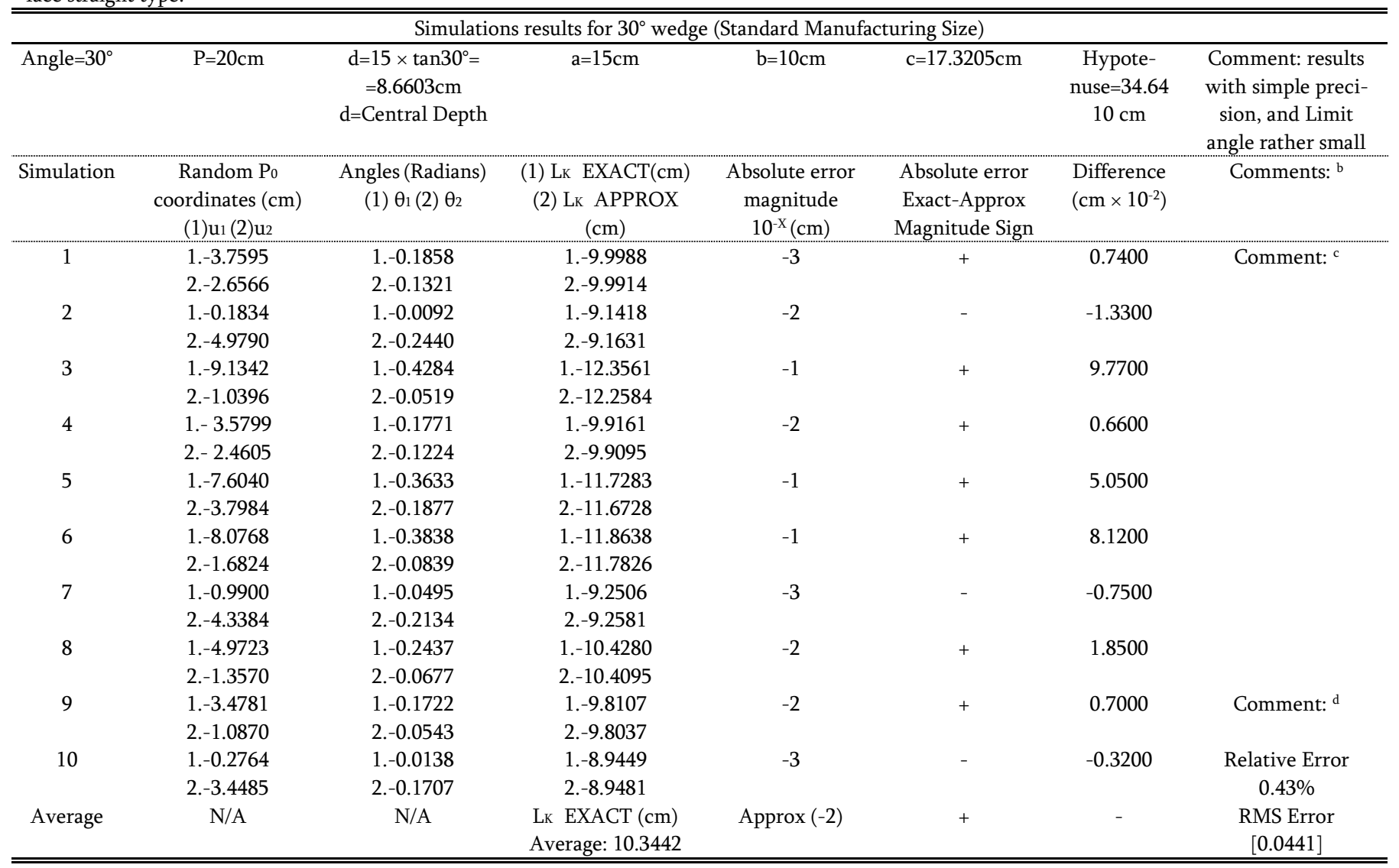

Comments: ${ }^{\mathrm{b}}=$ In general, when the wedge angle is small we find lower RMS Errors. Simulations ranges to avoid Limit Angle, u1 $\epsilon$ [0, 10] and u2 $\epsilon[0,5] ;{ }^{c}=$ All these value shows small differences, probably not exceeding the limit angle, at the thick part of the wedge; ${ }^{\mathrm{d}}=$ High precision in approximations, very low relative error. 
TABLE 3: $45^{\circ}$ Wedge filter angles. Simulation carried out at positive quadrant of the broad part of the wedge. Selected wedges are sloping surface straight type.

\begin{tabular}{|c|c|c|c|c|c|c|c|}
\hline \multicolumn{8}{|c|}{ Simulations results for $45^{\circ}$ wedge (Standard Manufacturing Size) } \\
\hline Angle $=45^{\circ}$ & $\mathrm{P}=20 \mathrm{~cm}$ & $\begin{array}{c}\mathrm{d}=15 \times \tan 45^{\circ}= \\
=15 \mathrm{~cm} \\
\mathrm{~d}=\text { Central Depth }\end{array}$ & $\mathrm{a}=15 \mathrm{~cm}$ & $\mathrm{~b}=10 \mathrm{~cm}$ & $\mathrm{c}=30 \mathrm{~cm}$ & $\begin{array}{c}\text { Hypote- } \\
\text { nuse }=42.462 \\
4 \mathrm{~cm}\end{array}$ & $\begin{array}{l}\text { Comment: re- } \\
\text { sults with simple } \\
\text { precision }\end{array}$ \\
\hline Simulation & $\begin{array}{c}\text { Random } \mathrm{P}_{0} \\
\text { coordinates } \\
(\mathrm{cm}) \\
(1) \mathrm{u}_{1}(2) \mathrm{u}_{2}\end{array}$ & $\begin{array}{l}\text { Angles (Radians) } \\
\text { (1) } \theta_{1}(2) \theta_{2}\end{array}$ & $\begin{array}{c}\text { (1) Lk EXACT }(\mathrm{cm}) \\
(2) \mathrm{LK} \text { APPROX } \\
(\mathrm{cm})\end{array}$ & $\begin{array}{l}\text { Absolute } \\
\text { error magni- } \\
\text { tude } 10^{-\mathrm{X}}(\mathrm{cm})\end{array}$ & $\begin{array}{l}\text { Absolute } \\
\text { error Exact- } \\
\text { Approx Mag- } \\
\text { nitude Sign }\end{array}$ & $\begin{array}{l}\text { Difference } \\
(\mathrm{cm})\end{array}$ & Comments: ${ }^{\mathrm{e}}$ \\
\hline \multirow[t]{2}{*}{1} & $1 .-5.6392$ & $1 .-0.2748$ & $1 .-20.8923$ & -1 & + & 0.2082 & \\
\hline & 2. -5.3133 & 2. -0.2597 & 2.-20.6841 & & & & \\
\hline \multirow[t]{2}{*}{2} & 1. -0.2751 & 1. -0.0138 & 1.-15.2995 & -1 & - & -0.0221 & \\
\hline & 2. -6.8971 & 2. -0.3321 & 2.-15.3216 & & & & \\
\hline \multirow[t]{2}{*}{3} & 1.-13.7013 & $1 .-0.6006$ & $1 .-53.0567$ & +2 & + & 3.7687 & Comment: ${ }^{f}$ \\
\hline & 2. -9.9580 & 2. -0.4620 & 2. -49.2880 & & & & \\
\hline \multirow[t]{2}{*}{4} & 1.- 5.3698 & 1. -0.2623 & 1.-20.5059 & -1 & + & 0.1724 & \\
\hline & 2.- 2.0792 & 2. -0.1036 & 2.- -20.3335 & & & & \\
\hline \multirow[t]{2}{*}{5} & $1 .-11.4060$ & 1.- -0.5183 & 1. -36.1161 & +1 & + & 4.5121 & \\
\hline & 2.-4.9211 & 2. -0.2413 & 2.- -31.6040 & & & & \\
\hline \multirow[t]{2}{*}{6} & 1.-12.1152 & 1. -0.5446 & 1. -40.1691 & +2 & + & 6.4374 & \\
\hline & 2.-7.5968 & 2. -0.3630 & 2.- -33.7317 & & & & \\
\hline \multirow[t]{2}{*}{7} & 1.- -1.4851 & 1.- -0.0741 & 1. -16.2177 & -3 & + & 0.0013 & \\
\hline & 2. -3.3648 & 2. -0.1669 & 2.- -16.2164 & & & & \\
\hline \multirow[t]{2}{*}{8} & 1.-7.4585 & 1. -0.3570 & 1. -24.0277 & +1 & + & 0.6671 & \\
\hline & 2. -8.6696 & 2. -0.4090 & 2. -23.3606 & & & & \\
\hline \multirow[t]{2}{*}{9} & $1 .-5.2172$ & 1.- -0.2552 & 1. -20.2948 & -1 & + & 0.1550 & \\
\hline & 2. -2.7140 & 2. -0.1349 & 2. -20.1398 & & & & \\
\hline \multirow[t]{2}{*}{10} & 1.- 0.4147 & 1.- -0.0207 & 1. -15.3259 & -3 & - & -0.0001 & Relative Error \\
\hline & 2. -2.1740 & 2. -0.1083 & 2. -15.3260 & & & & $10.6 \%$ \\
\hline \multirow[t]{2}{*}{ Average } & N/A & N/A & LK EXACT $(\mathrm{cm})$ & Approx $(-1)$ & + & - & RMS Error \\
\hline & & & Average:26.1906 & & & & {$[2.7667]$} \\
\hline
\end{tabular}

Comments: ${ }^{\mathrm{e}}=$ In general, when the wedge angle increases we find bigger RMS Errors; ${ }^{\mathrm{f}}=$ This value shows large differences, at the edge of the wedge 
TABLE 4: $\left(60^{\circ}\right.$ Wedge filter angles. Simulation carried out at positive quadrant of the broad part of the wedge. selected wedges are sloping surface straight type)

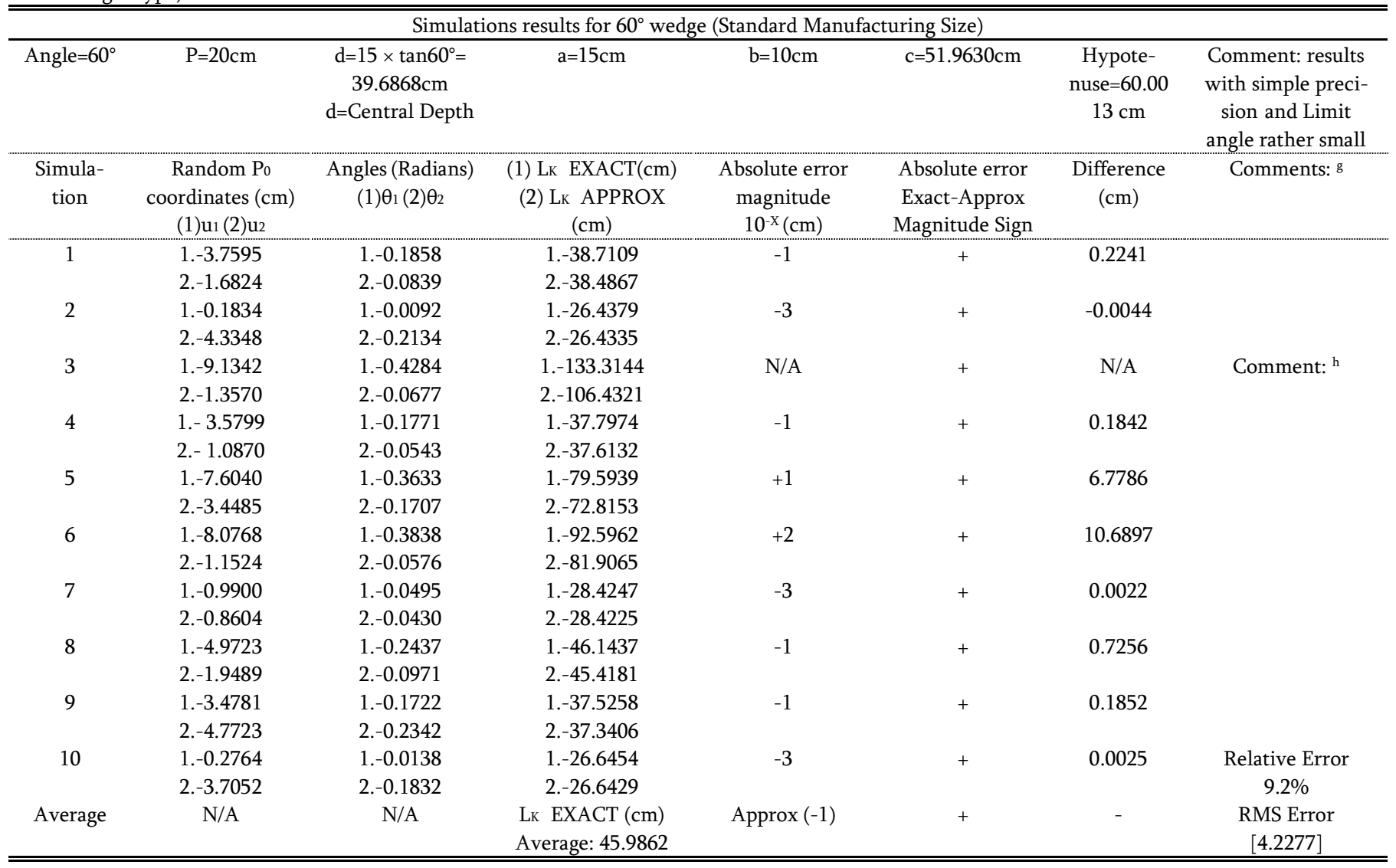

Comments: ${ }^{\mathrm{g}}=$ In general, when the wedge angle increases we find bigger RMS Errors. Simulations ranges to avoid limit angle, u1 $\epsilon$ [0, 10] u2 $\epsilon$ [0, $5] ;{ }^{\mathrm{h}}=$ This value shows large differences, probably exceeding limit angle, then it is discarded. 